\title{
Use of polydispersity index as control parameter to study melting/freezing of Lennard-Jones system: Comparison among predictions of bifurcation theory with Lindemann criterion, inherent structure analysis and Hansen-Verlet rule
}

\author{
SARMISTHA SARKAR ${ }^{\mathrm{a}, \mathrm{b}}$, RAJIB BISWAS ${ }^{\mathrm{a}}$, PARTHA PRATIM RAY ${ }^{\mathrm{b}}$ and BIMAN BAGCHI ${ }^{\mathrm{a}, *}$ \\ ${ }^{a}$ Solid State and Structural Chemistry Unit, Indian Institute of Science, Bangalore 560 012, India \\ ${ }^{\mathrm{b}}$ Department of Physics, Jadavpur University, Kolkata 700 032, India \\ e-mail: bbagchi@sscu.iisc.ernet.in; profbiman@gmail.com
}

MS received 2 April 2015; revised 6 June 2015; accepted 2 July 2015

\begin{abstract}
Using polydispersity index as an additional order parameter we investigate freezing/melting transition of Lennard-Jones polydisperse systems (with Gaussian polydispersity in size), especially to gain insight into the origin of the terminal polydispersity. The average inherent structure (IS) energy and root mean square displacement (RMSD) of the solid before melting both exhibit quite similar polydispersity dependence including a discontinuity at solid-liquid transition point. Lindemann ratio, obtained from RMSD, is found to be dependent on temperature. At a given number density, there exists a value of polydispersity index $\left(\delta_{\mathrm{P}}\right)$ above which no crystalline solid is stable. This transition value of polydispersity (termed as transition polydispersity, $\delta_{\mathrm{P}}$ ) is found to depend strongly on temperature, a feature missed in hard sphere model systems. Additionally, for a particular temperature when number density is increased, $\delta_{\mathrm{P}}$ shifts to higher values. This temperature and number density dependent value of $\delta_{\mathrm{P}}$ saturates surprisingly to a value which is found to be nearly the same for all temperatures, known as terminal polydispersity $\left(\delta_{\mathrm{TP}}\right)$. This value $\left(\delta_{\mathrm{TP}} \sim 0.11\right)$ is in excellent agreement with the experimental value of 0.12 , but differs from hard sphere transition where this limiting value is only 0.048 . Terminal polydispersity $\left(\delta_{\mathrm{TP}}\right)$ thus has a quasiuniversal character. Interestingly, the bifurcation diagram obtained from non-linear integral equation theories of freezing seems to provide an explanation of the existence of unique terminal polydispersity in polydisperse systems. Global bond orientational order parameter is calculated to obtain further insights into mechanism for melting.
\end{abstract}

Keywords. polydispersity index; Lindemann ratio; Hansen-Verlet rule; bifurcation diagram; terminal polydispersity; inherent structure energy

\section{Introduction}

Although melting and freezing ${ }^{1-4}$ are thermodynamically equivalent, the molecular mechanism of phase change and the respective theories are vastly different for the two cases. In this work we consider two criteria for melting (Lindemann criterion ${ }^{5}$ of melting and inherent structure ${ }^{6}$ analysis) and two criteria for freezing (Hansen Verlet rule $^{7}$ for the nearly constant value of the first peak of the liquid static structure factor at freezing and the integral equation theory of freezing). ${ }^{8,9}$ All these criteria are used to investigate the agreement or lack of it among the different approaches. Inherent structure (IS) analysis to melting has not been employed before in the present context.

Historically there have been two approaches to study melting/freezing. One is the instability based theories that do not use thermodynamic criteria such as equality of pressure and chemical potential. An example is

*For correspondence provided by Lindemann criterion of melting. Melting is associated with instability of solids. Similarly, in the liquid to solid transition, the study of liquid instability has been pursued by Lovett, ${ }^{10}$ Munakata ${ }^{11,12}$ and many others. In this approach, the freezing is associated with an instability of the liquid to periodic density waves of wave number $\mathrm{k}_{\mathrm{n}}$ where the static structure exhibits the first peak. It is not known whether the value obtained for temperature or density is the same when the two transitions occur, according to these semi-phenomenological criteria. Thus, the values of relevant transition parameters obtained from the two phase transitions, although equivalent thermodynamically, might not be equal when viewed through instability conditions. It is known that while a liquid can be super cooled considerably, the same is not true for a solid. To the best of our knowledge, there has been no critical analysis of the agreement/ disagreement of the criteria mentioned above. This can be studied more conveniently in polydisperse system by using polydispersity index as a control parameter. 
When the value of polydispersity index $(\delta)$ goes beyond a critical value, then liquid does not transform into solid if we come from liquid side. In both the transitions we can identify the critical value of $\delta$ which in principle need not be the same unless and until equality of the critical potential (at constant pressure) is employed.

In fact, the effect of polydispersity presents an interesting case study in the solid-liquid phase transition of Lennard-Jones polydisperse system. In a polydisperse liquid or solid, each atom/molecule can be different and distinguishable, unlike in a one component system. With the increase in polydispersity, the energy-entropy balance becomes increasingly unfavorable for the solid to exist as a stable phase. In the polydisperse system, the energy of the solid increases due to building up of strain energy owing to increasing mismatch in size of the neighbours, while the entropy of the liquid increases. These two factors lead to the existence of a terminal polydispersity beyond which the solid phase cannot be stabilized. Thus, polydispersity index serves as an additional order parameter of the system. Several earlier studies proposed that homogeneous crystallization never takes place beyond an upper limit on the size polydispersity and it is surprisingly independent of pressure $\left(\mathrm{P}^{*}\right)$ and temperature $\left(\mathrm{T}^{*}\right)$ and number density $(\rho)$. This value of polydispersity index is sometimes termed as "terminal polydispersity" above which the freezing transition ${ }^{1,2,4,13-19}$ is not possible.

In an earlier study, analysis of the nonlinear integral equations for inhomogeneous density (corresponding to freezing of the fcc) led to the development of bifurcation theory of freezing, as developed by Kozak et al. ${ }^{9}$ This theory suggested the existence of a terminal liquid density that could be identified with the random close packed (RCP) state. The coincidence of density at the terminal polydispersity with the RCP state bears a similarity to the analysis of Rice et al. ${ }^{20}$ for a hard sphere system. A similar analysis of Lennard-Jones (LJ) system ${ }^{21}$ was carried out by the same group. Phan et al. ${ }^{4}$ showed that for Lennard-Jones potential, the density of RCP state was weakly temperature dependent.

There have been several discussions ${ }^{3,22,23}$ that address the limit of freezing. While Lovett ${ }^{24}$ considered this as a spinodal decomposition, ${ }^{25}$ Bagchi-Cerjan-Rice suggested that it was the last point of the liquid state where the liquid could transform to solid. Beyond this point no solution to integral equation theory was available. In another approach ${ }^{26,27}$ that considered the pair entropy as a freezing criterion of Morse and Lennard-Jones liquids, the pair correlation entropy of these simple liquids was shown to have a threshold value at freezing. Some of the recent works by Dyre and co-workers ${ }^{28}$ on mapping of simple liquids to inverse power law potentials and isomorph picture of simple liquids have proven to be rather useful in understanding the common basis of single phase melting and freezing rules. Polydisperse systems and their freezing behavior provide an interesting test of these ideas.

From earlier studies on the freezing of polydisperse fluids, a fascinating outcome was the observation of microscopic fractionation, ${ }^{29}$ in which local polydispersity could be different among the coexisting phases. Another fascinating observation was the reentrant melting ${ }^{30,31}$ that had been observed for hard spheres at large polydispersity and large volume fraction. In these limits, (where pressure was also high) the disordered state was more stable. Recently, an application of density functional theory (DFT) of freezing ${ }^{32,33}$ of hard sphere fluid by Chaudhuri et al., found a terminal polydispersity of 0.048 , followed by a reentrant melting at larger density. ${ }^{34}$ Wolynes and coworkers. ${ }^{35}$ had demonstrated that DFT could provide a useful theoretical framework for analyzing the freezing of a supercooled liquid into a large number of disordered structures.

In our earlier work, ${ }^{1}$ we have used inherent structure ${ }^{6}$ (IS) as an insightful parameter to study solid-liquid transition of Lennard-Jones polydisperse system. Inherent structure $^{36}$ are obtained by removing the kinetic energy, including vibrations, of the atoms and molecules comprising the system. We have shown that beyond the terminal polydispersity, crystalline solid cannot be obtained in the IS and the transition line does not show the signature of reentrant melting. This absence of reentrant melting in the Lennard-Jones polydisperse system may be attributed to the influence of the attractive part of the potential upon melting, but needs to be verified in a more systematic study.

In this article, we have applied both theory and computer simulation to understand the origin of terminal polydispersity in the solid-liquid transition in LennardJones polydisperse spheres. Here we assume that our polydisperse system consists of particles having different sizes, sampled from a Guassian distribution. To this purpose, we use integral equation theory of freezing and attempt to correlate the bifurcation diagram to the existence of terminal polydispersity.

In solid-liquid transition, when molecules/atoms interact with each other via a central potential, two empirical criteria have been used extensively to predict transitions. From the melting side one uses the Lindemann criterion $^{5}$ which states that at melting temperature, the root mean square displacement exceeds 0.1 of the cell length. The second criterion is the Hansen-Verlet rule of crystallization $^{7}$ that requires the value of the first peak of static structure factor $\mathrm{S}(\mathrm{k})$ to exceed 2.85 for freezing. 
Both these simple criteria have been found to hold good for different types of interactions. Lindemann criteria of melting can be investigated using lattice dynamical theory. On the other hand, justification of the Hansen-Verlet rule of crystallization comes from the density functional theory of freezing $32,33,35,37-40$

We find the Lindemann ratio (L) obtained from root mean square displacement (RMSD) to be rather strongly dependent on temperature. We explore the correlation between RMSD (related to Lindemann parameter) and IS showing almost the same kind of nature with respect to different degrees of polydispersity. Our study reveals that the transition polydispersity $\left(\delta_{\mathrm{P}}\right)$ converges to a maximum value of 0.11 , termed as terminal polydispersity $\left(\delta_{\mathrm{TP}}\right)$ that is in agreement with the experimental value $^{41}$ of 0.12 . We use separately two well-known criteria of melting/freezing, the Lindemann criterion of melting and the Hansen-Verlet rule of freezing, to predict terminal polydispersity and these predictions ${ }^{5,7}$ are also in good agreement with each other.

Pusey ${ }^{42}$ proposed a simple criterion for the terminal polydispersity based on an analogy of the Lindemann melting criterion; he also obtained a value of about $11 \%$. Moreover, Pusey ${ }^{43}$ performed experiments in which he observed that dispersions with a polydispersity of $7.5 \%$ would (partly) freeze, while those with a polydispersity of $12 \%$ did not. Chakravarty, Debenedetti and Stillinger ${ }^{44}$ looked at the Lindemann ratio within an inherent structure framework to understand the solidliquid phase transition in a Lennard-Jones-type system. A useful summary for the properties of coexisting fluid and solid phases in the case of soft sphere is given by Kofke and co-workers. ${ }^{45}$ Several semi-empirical 'melting rules' were examined by Kofke et al. in the light of the results. Moreover, Bolhuis and kofke ${ }^{19}$ have shown the existence of terminal polydispersity for the solid phase to be $5.7 \%$ and for the liquid phase to be $11.8 \%$ that is also in good agreement with our present result.

In fact, the existence of terminal polydispersity is interesting in the study of freezing/melting transition, ${ }^{35,37-39,46-54}$ and to the best of our knowledge, not fully understood.

In our present study, we examine several aspects of the Lennard-Jones polydisperse system in order to achieve a better understanding of the effects of polydispersity on the freezing-melting transition. In essence, we look for the following issues and questions in this study:

1. The behaviour of the Lennard-Jones polydisperse system is analysed from three distinct ways, namely, by applying the Lindemann Criterion of melting, the inherent structure analysis and the empirical Hansen-Verlet rule of freezing. The results thus obtained are in good agreement with one another in predicting the existence of a terminal polydispersity, beyond which liquid-solid transition is not possible.

2. These findings are interpreted in terms of the theory of freezing ${ }^{20}$ developed by Bagchi, Cerjan, and Rice using bifurcation diagram. We have discussed how certain features of the scaled phase diagram may be used to explain the terminal polydispersity in polydisperse system.

3. A set of Lindemann measures, based on RMSD, is applied to understand solid-liquid transition.

4. IS analysis has been used to predict transition and terminal polydispersity. The correlation between IS and RMSD, with respect to different degree of polydispersity, is explored.

5. Temperature is a formidable effect and hence it deserves detailed study. Most of the earlier studies are based on hard sphere system where attractive interaction potential is not taken into account and it shows reentrant melting. Hard sphere system does not allow to explore the temperature effect on transition. On the other hand, in the present Lennard-Jones model system, it is interesting to study the temperature dependence of transition and the influence of the attractive part of potential on the terminal polydispersity.

The rest of the paper is organized as follows. In Sec. 2, we present the details of the simulations. In Sec. 3, we organize the results in different subsections and we illustrate theoretical background of the study. In Sec. 3.6, we discuss our results. In Sec. 4, we present the concluding remarks on the work.

\section{Simulation details}

\subsection{Molecular dynamics (MD) and Monte Carlo (MC) simulations}

We study all simulations using standard Molecular dynamics (MD) and Monte Carlo (MC) techniques supplemented by a particle swap algorithm. It is well known that standard simulation method is not completely capable of locating the true thermodynamic equilibrium of the system. Hence the particle swap technique improves this drawback to some extent. Our system consists of $\mathrm{N}=500$ particles in a periodically repeated cubic box with volume $\mathrm{V}$ having Gaussian distribution of particle diameters $\sigma$,

$$
P(\sigma)=\frac{1}{\sqrt{2 \pi d^{2}}} \exp \left[-\frac{1}{2}\left(\frac{\sigma-\bar{\sigma}}{d}\right)^{2}\right] .
$$

The dimensionless polydispersity index is defined as $\delta=\frac{d}{\bar{\sigma}}$, where $\mathrm{d}$ is standard deviation of the distribution 
and $\bar{\sigma}$ is the mean diameter. The number density is $\rho=N / V$. The particles interact with LJ potential with a constant potential depth. The potential for a pair of particles $i$ and $j$ is cut and shifted to zero, at distance $r_{c}=$ $2.5 \sigma_{i j}$, where $\sigma_{i j}=\left(\sigma_{i}+\sigma_{j}\right) / 2$. We study for different polydispersity indices starting from $\delta=0$ up to $\delta=0.20$ with an increment of 0.01 . Both MD and MC simulations have been employed in NVT, NPT, and NVE ensembles. The MC simulations are aided with particle swapping to enable a faster equilibration in the solid phase. MC simulations with larger system size (2048 particles) are carried out to check the finite size effects and the overall physical picture remains the same upon variation of system size.

\subsection{Computing inherent structures}

The configurations of the system corresponding to the local potential energy minima, known as the inherent structure (IS), are obtained from computer simulations. Inherent structures are identified in the simulation by a steepest descent minimization of the potential energy. The standard conjugate gradient method ${ }^{55}$ is employed to obtain the IS along an MD trajectory from which the average IS energy is computed corresponding to different polydispersity indices.

\subsection{Calculation of transition and terminal polydisper- sity (as shown in the forthcoming sections 3.1 and 3.2)}

The transition and terminal polydispersity at different temperatures are calculated in the following manner. For each polydispersity we perform simulation in NVT ensemble for 50000 steps, followed by 50000 steps for equilibration in the NVE ensemble and 500000 more steps in the NVE ensemble to obtain results. The single time step taken is $2 \mathrm{fs}$. Corresponding to a given temperature for the parent liquid, IS energy increases almost quadratically with increasing polydispersity index up to a certain value (at which structural transition happens) that is termed as transition polydispersity $\left(\delta_{\mathrm{P}}\right)$ for a particular volume fraction. We calculate density from the corresponding volume fraction using the relation $\rho=$ $6 \Phi / \pi$ to plot density versus transition polydispersity curve. With increasing density $(\rho), \delta_{\mathrm{P}}$ increases and ultimately converges to a constant, temperature independent value which is termed as the "terminal polydispersity" $\left(\delta_{\mathrm{TP}}\right)$ of the system.

\subsection{Capturing melting mechanism (as shown in the forthcoming section 3.4)}

In order to capture the mechanism of melting we present the simulation study on the NPT ensemble of the polydisperse system having 1372 particles at polydispersity index $\delta=0.08$. Same procedure is repeated for different polydispersity indices with different system sizes. Using NPT simulation, temperature is gradually increased starting from $\mathrm{T}^{*}=0.50$ with an increment of 0.04 until the solid is melted. We compute global bond order to capture the melting mechanism more clearly.

\section{Results and Discussion}

\subsection{Correlation between average inherent structure energy and root mean square displacement during solid- liquid transition}

With the increase in polydispersity indices, average IS energy as well as RMSD increases because of the increasing frustration of the system. Same trend can be visualized for both the cases. Predictions of transition polydispersity from IS energy analysis and RMSD are in good agreement with each other. This shows that there is a strong correlation between average inherent structure energy and RMSD as discussed below.

\section{1a Root mean square displacement and Lindemann} criterion of melting in polydisperse system - Prediction of transition polydispersity: The root mean square displacement is connected to the Lindemann parameter that is widely used for the prediction of the melting temperature of a variety of solids. This phenomenological criterion was put forward by Lindemann in 1910 . The idea behind the theory was the observation that the average amplitude of thermal vibrations increases with increasing temperature. ${ }^{5}$ Melting initiates when the amplitude of vibration becomes large enough for adjacent atoms to partly occupy the same space. The Lindemann criterion states that melting is expected when the root mean square vibration amplitude exceeds a threshold value. Hence one can write,

$$
L=\frac{\sqrt{\left\langle\vec{r}_{i}^{2}\right\rangle}}{a},
$$

where $L$ is the Lindemann parameter for the associated polydisperse system and $a$ is the mean distance between the particles. This threshold value of Lindemann constant is $L \approx 0.1$ for melting of crystals ${ }^{56}$ of point-like particles in three dimensions, but it may vary between 0.05 and 0.20 depending on different factors like nature of inter particle interactions, crystal structure, and magnitude of quantum effects.

Figure 1 shows the variation of amplitude of RMSD with respect to polydispersity indices $(\delta)$ for two different temperatures $\mathrm{T}^{*}=1.0$ and 0.5 for a constant volume fraction $\Phi=0.58$. The plot shows that the value of 


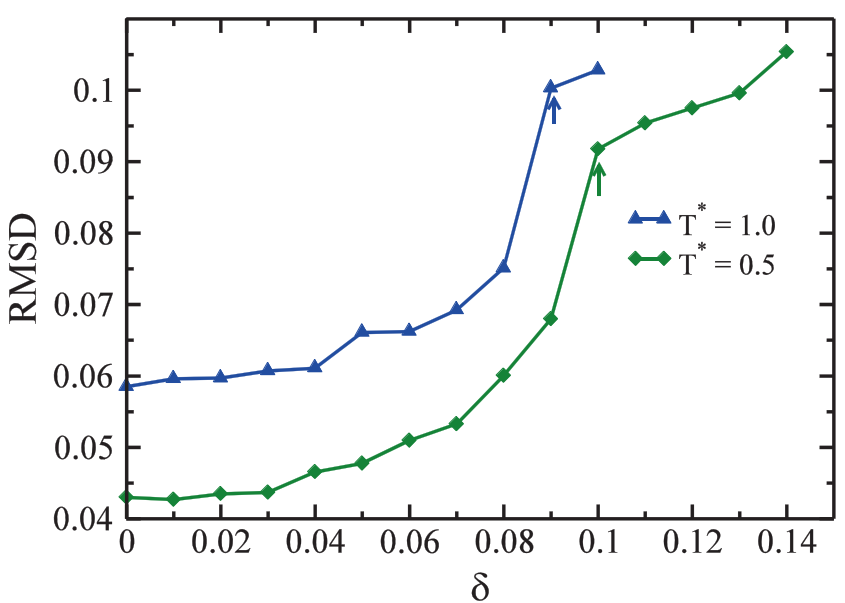

Figure 1. Variation of root mean square displacement with respect to polydispersity indices $(\delta)$ for two different temperatures $T^{*}=1.0$ (blue solid line with filled triangle up) and 0.5 (green solid line with filled diamond) for a constant volume fraction $\Phi=0.58$. Note that with increase in $\delta$, the value of RMSD also increases. For temperature $T^{*}=0.10$, a large change in the slope occurs at $\delta=0.09$ (as indicated by blue arrow) and for $T^{*}=0.5$, the same happens at $\delta=0.10$ (as indicated by green arrow). Note that blue and green arrows signify the onset of melting process at $\delta=0.09$ and 0.10 corresponding to the temperatures $T^{*}=1.0$ and 0.5 respectively. Lindemann ratio (related to RMSD) is taken as $\mathrm{L}=$ 0.1 for $T^{*}=1.0$ and $\mathrm{L}=0.09$ for $T^{*}=0.5$. Note that Lindemann ratio (L), obtained from RMSD, is not a universal number ( 0.1 of cell length), but it is found to be dependent on temperature, as shown in the figure.

RMSD increases with the increase of $\delta$. For temperatures $T^{*}=1.0$ and 0.5 , the slope of the curve changes sharply at $\delta=0.09$ and 0.10 respectively. This signifies the onset of melting processes at $\delta=0.09$ for $T^{*}=1.0$ and $\delta=0.10$ for 0.5 in the Lennard-Jones polydisperse solid, when the amplitude of the root mean square vibration exceeds a threshold value of Lindemann ratio taken as $\mathrm{L}=0.1$ for $T^{*}=1.0$ and $\mathrm{L}=0.09$ for $T^{*}=0.5$. Lindemann ratio $(\mathrm{L})$, obtained from RMSD, is found to be strongly dependent on temperature.

\section{1b Prediction of transition polydispersity from inher-} ent structure analysis: As shown in figure 2(a), the average IS energy is computed with respect to polydispersity index ( $\delta$ ) at constant $T^{*}=1.0$ for $\Phi=0.58$. Figure 2(b) shows the average IS energy with respect to different volume fractions $\Phi=0.48,0.52,0.58,0.64,0.70$ at the same temperature $T^{*}=1.0$. In both the cases, as shown in figure 2(a) and figure 2(b), the average IS energy increases almost quadratically with polydispersity up to a polydispersity index at which structural transition takes place and the plots change their corresponding slopes. This polydispersity index is known as transition polydispersity $\left(\delta_{\mathrm{P}}\right)$ beyond which the crystalline solid
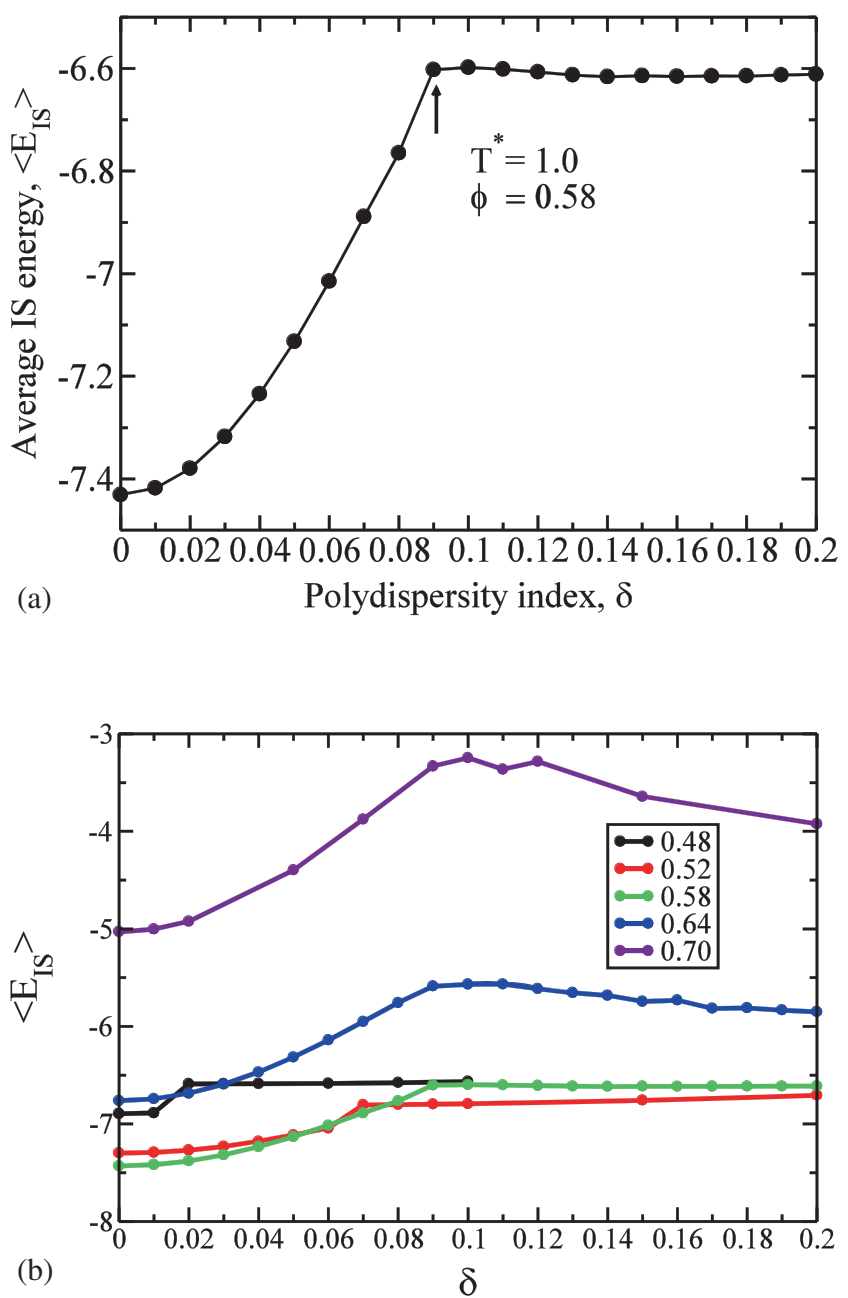

Figure 2. (a) Variation of average IS energy with respect to polydispersity index $(\delta)$ until transition polydispersity $\left(\delta_{\mathrm{P}}\right)$ at constant temperature $T^{*}=1.0$ for $\Phi=0.58$. Note that the black arrow signifies transition polydispersity $\left(\delta_{\mathrm{P}}\right)$ indicating the point of structural transition where the dependence of average IS energy with $\delta$ changes sharply. (b) Variation of average IS energy with respect to $\delta$ at constant temperature $T^{*}=1.0$ for different volume fractions $\Phi=0.48$, $0.52,0.58,0.64,0.70$. The average IS energy increases with increase in $\delta$ until transition polydispersity $\left(\delta_{\mathrm{P}}\right)$ when the dependence changes sharply. Note that at low volume fraction $\Phi$, the transition occurs at lower value of $\delta$ and at higher $\Phi$, solid phase is stable up to higher $\delta$ value.

phase is no longer stable and amorphous phase exists. The transition occurs at lower value of $\delta$ for lower $\Phi$ and solid phase is stable up to higher $\delta$ value for higher $\Phi$.

We achieve the transition polydispersity $\left(\delta_{\mathrm{P}}\right)$ for different volume fractions using IS analysis. The crossover of the two slopes corresponding to each $\Phi$ signifies the transition polydispersity where the structural transition from solid to amorphous state takes place. Table 1 shows the transition polydispersities $\left(\delta_{\mathrm{P}}\right)$ for the melting transition of the Lennard-Jones polydisperse system 
Table 1. Transition polydispersity $\left(\delta_{\mathrm{P}}\right)$ for the melting transition of Lennard-Jones polydisperse systems predicted from IS analysis. The first column represents the volume fraction $\Phi$ and second column represents corresponding $\delta_{\mathrm{P}}$ at constant $\mathrm{T}^{*}=1.0$.

\begin{tabular}{lc}
\hline$\Phi$ & $\delta_{\mathrm{P}}$ \\
\hline 0.48 & 0.02 \\
0.52 & 0.07 \\
0.58 & 0.09 \\
0.64 & 0.10 \\
0.70 & 0.11 \\
\hline
\end{tabular}

corresponding to different values of volume fractions at constant $\mathrm{T}^{*}=1.0$.

Difference in the polydispersity index $(\delta)$ dependence of the inherent structure (IS) energy in the crystalline solid and in the amorphous phase is understandable in terms of the theory of elasticity in solids. ${ }^{57}$ In the presence of a strain field, the free energy of a body can be written as,

$$
F=F_{0}+\frac{1}{2} \lambda u_{i i}^{2}+\mu u_{i k}^{2},
$$

where $\lambda$ and $\mu$ are called Lamé coefficients.

$$
K=\lambda+\frac{2}{3} \mu \text {. }
$$

Here $\mathrm{K}$ and $\mu$ are bulk modulus and modulus of rigidity respectively.

Now in the crystalline solid, polydispersity introduces strain field $u_{i k}$ which is randomly distributed in the solid and strongly dependent on $\delta$. As $\delta$ increases, strain field also increases. The quantitative estimate in the free energy can be achieved from the densityfunctional theory of elasticity. ${ }^{58,59}$ On the other hand, the particles in the amorphous phase can undergo rearrangements to reduce the strain which explains the weak dependence of the IS energy with $\delta$ in the amorphous phase. This allows us to estimate quantitatively the criterion of transition from IS analysis. Another point to notice is that the change of the IS energy at the cross over depends both on temperature and volume fraction. As the volume fraction is lowered, the effect of the strain field on the IS energy of the solid decreases. This describes the reason of different transition polydispersity indices $\left(\delta_{\mathrm{P}}\right)$ for different volume fractions.

\subsection{Quenched Phase diagram of the system}

For a particular number density $(\rho)$ and temperature $\left(\mathrm{T}^{*}\right)$, there is a value of polydispersity index, when the system undergoes melting transition from the solid state to amorphous state, known as "transition polydispersity"

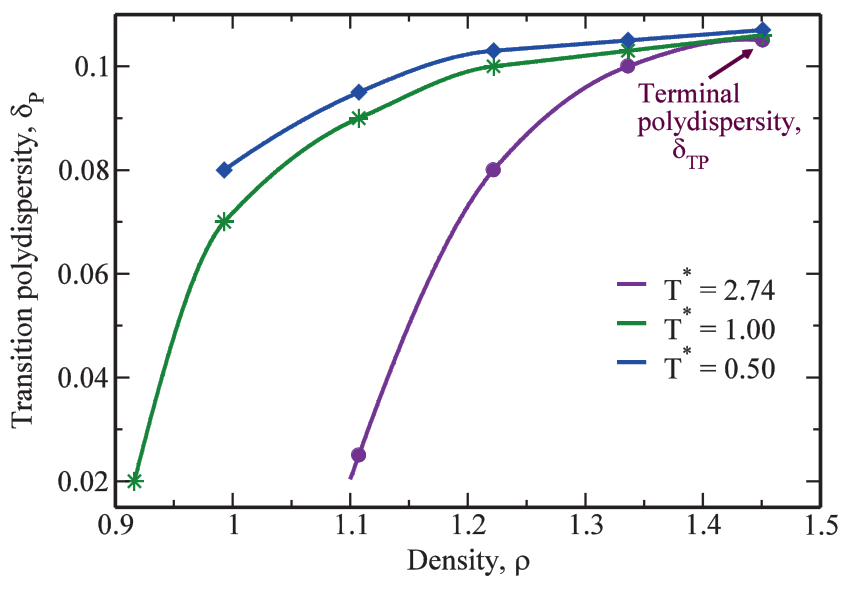

Figure 3. The transition polydispersity $\left(\delta_{\mathrm{P}}\right)$ increases with increasing number density $(\rho)$ at three different temperatures $\mathrm{T}^{*}=0.50$ (blue solid line with filled diamond), 1.00 (green solid line with star), 2.74 (violet solid line with filled circle). Transition polydispersity $\left(\delta_{\mathrm{P}}\right)$ becomes invariant of $\mathrm{T}^{*}$ and $\rho$ at $\delta_{\mathrm{TP}} \sim 0.11$. No liquid-solid transition is possible above this value and is known as "terminal polydispersity". Note that the existence of a nearly temperature invariant terminal polydispersity $\left(\delta_{\mathrm{TP}} \sim 0.11\right)$ comes from inherent structure analysis, that reveals the absence of crystallization at large $\delta$ values. The inherent structure analysis also finds a sharp transition between ordered and disordered states.

$\left(\delta_{\mathrm{P}}\right)$. Initially $\delta_{\mathrm{P}}$ increases with increasing $\rho$ and then eventually converge to a constant, temperature independent value and it is termed as the "terminal polydispersity" $\left(\delta_{\mathrm{TP}}\right)$ of the system.

As shown in figure 3, the transition polydispersity, obtained from IS energy calculation, increases with increasing $\rho$ at three different temperatures $\mathrm{T}^{*}=0.50$, $1.00,2.74$. At large $\rho$, transition polydispersity becomes invariant of $\mathrm{T}^{*}$ and $\rho$. No liquid-solid transition is possible above this value $\left(\delta_{\mathrm{TP}} \sim 0.11\right)$, and is called terminal polydispersity. The transition line increases monotonically and subsequently becomes saturated at higher values of density $(\rho)$. It is evident from figure 3 that the transition line does not show any signature of reentrant melting.

\subsection{Structural patterns of parent structure and corre- sponding inherent structure at different polydispersity indices}

The transition polydispersity $\left(\delta_{\mathrm{P}}\right)$ for the melting transition of the Lennard-Jones polydisperse system needs further exploration. We study the structural aspects at different polydispersity indices. We categorize the particles into two sub ensembles; particles with diameter less than 1.0 are termed as small particle and rest of the particles as big particle. In figure 4 we represent the snapshots showing the spatial positions of small particle 
(a)

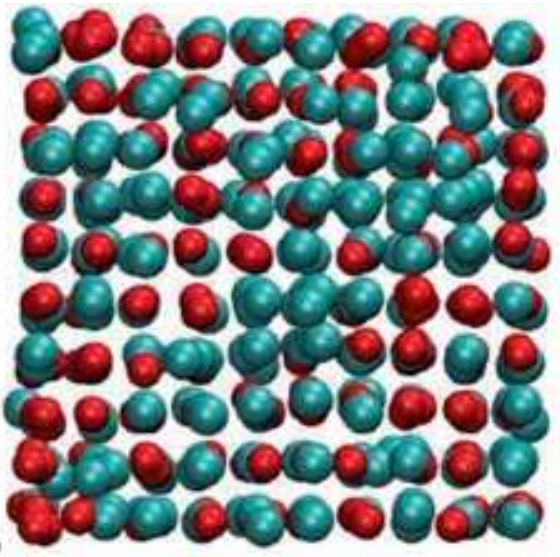

(c)

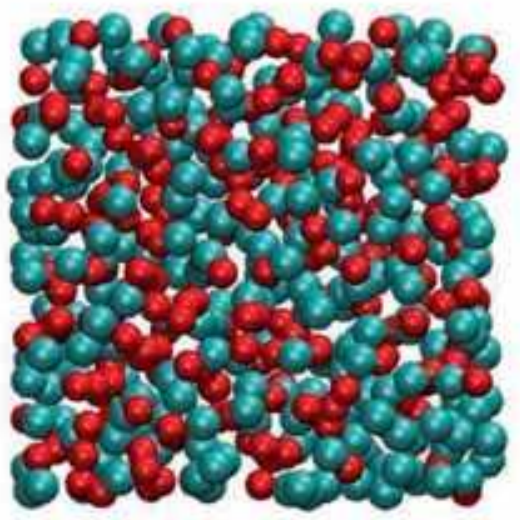

(b)

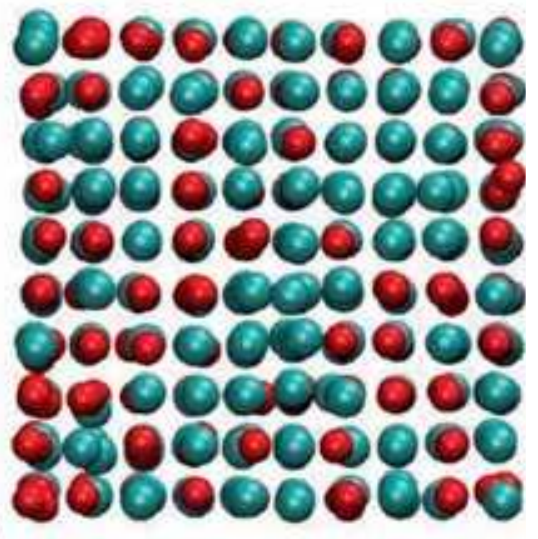

(d)

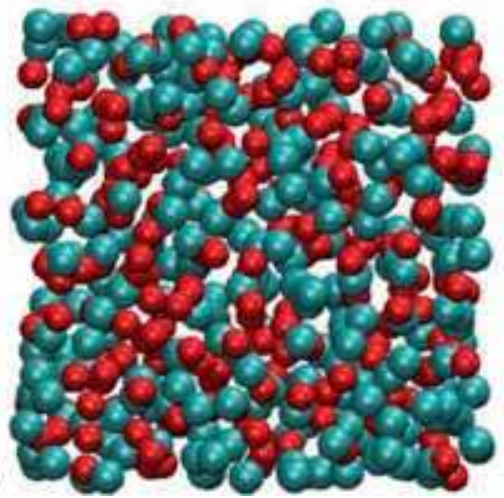

Figure 4. (Color online) Snapshots showing the spatial positions of small particle in red and big particle in cyan color in the parent structure along with its corresponding inherent structure at different polydispersity indices. Please note that the parent structure is at temperature $\mathrm{T}^{*}=1$ and $\Phi=0.58$. (a) Parent structure corresponds to the polydispersity index 0.08 . Note the crystal-like structure of the parent polydisperse system; (b) Inherent structure at $\delta=0.08$ shows the more structured formation than the corresponding parent structure; (c) Snapshot shows parent structure at $\delta=0.09$; and (d) corresponding inherent structure at $\delta=0.09$. Note that figure $4(\mathrm{c}, \mathrm{d})$ depict amorphous/glass like formation in the parent and inherent structure at $\delta=0.09$.

in red and big particle in cyan color in the parent structure along with its corresponding inherent structure at different polydispersity indices. The snapshots of the parent liquid have been taken at reduced temperature $\mathrm{T}^{*}=1.0$ and volume fraction $\Phi=0.58$. Figure $4(\mathrm{a})$ shows an instantaneous parent structure at $\delta=0.08$ and figure 4(b) shows its corresponding inherent structure while figure 4(c) and (d) depict molecular arrangements for the parent structure at $\delta=0.09$ and its inherent structure respectively. Figure 4(a) and (b) reveal the crystal-like arrangement of the particles in both parent and inherent structure respectively. Figure 4(c) and 4(d) show instantaneous parent structure and corresponding inherent structure at $\delta=0.09$ which seem to be almost homogeneous. These four snapshots show structural transition of the present L-J polydisperse system when it enters from $\delta=0.08$ to $\delta=0.09$ at $\mathrm{T}^{*}=1.0$.

\subsection{Insight into mechanisms of melting: Global bond orientational order parameter}

In this section, we study the temperature induced melting of the polydisperse system to provide insights into the mechanisms of melting. During NPT simulation, with constant $\mathrm{P}^{*}=10$, temperature is increased gradually starting from $\mathrm{T}^{*}=0.50$ with an increment of 0.04 for the polydisperse system at $\delta=0.08$. Figure 5(a) depicts number density $(\rho)$ as a function of temperature. Abrupt jump in the value of density signals melting as shown in figure 5(a) where upper part of densitytemperature curve denotes the solid branch ending at $\mathrm{T}^{*}=1.02$ and lower one denotes the liquid branch starting at $\mathrm{T}^{*}=1.06$ during solid-liquid transition.

One can use the local bond order introduced by Steinhardt et $a l .{ }^{60}$ to quantify the variation in the amplitude of structural change with polydispersity. 

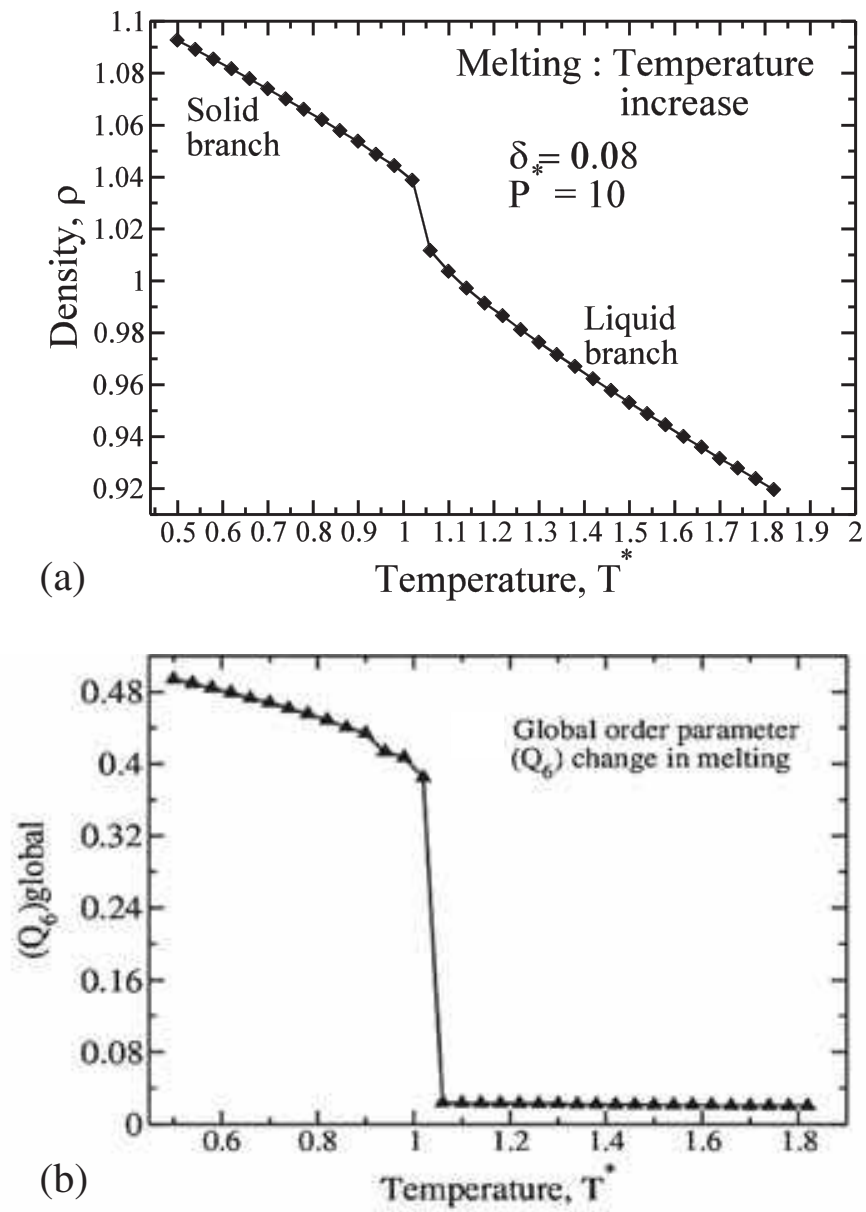

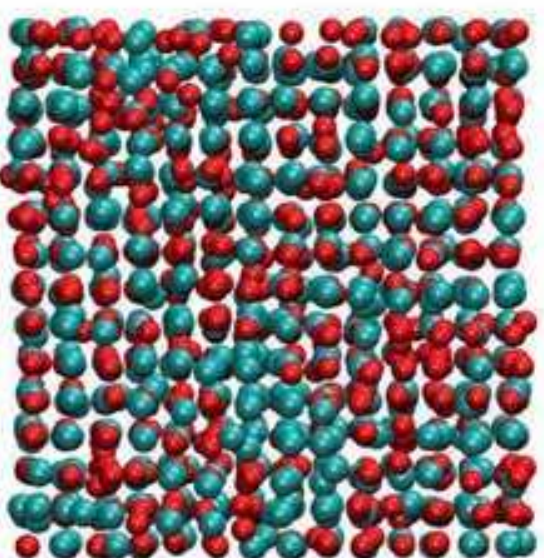

(c)

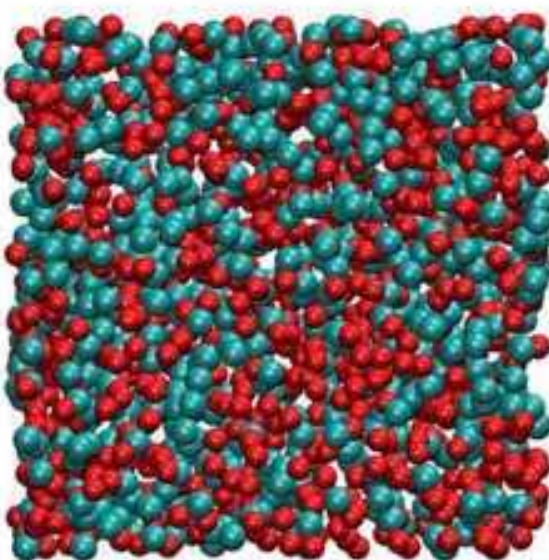

(d)

Figure 5. (a) Number density $(\rho)$ as a function of temperature $\mathrm{T}^{*}$ at $\delta=0.08$ and constant $\mathrm{P}^{*}=10$. Abrupt jump in density shows the signature of solid-liquid transition. Please note that upper part of density-temperature curve denotes solid branch ending at $\mathrm{T}^{*}=1.02$ and lower part denotes liquid branch starting at $\mathrm{T}^{*}=1.06$. (b) Change of global order parameter $\left(\mathrm{Q}_{6}\right)$ during melting of the solid. The plot confirms the first-order nature of phase transition indicated by a sharp fall in $\mathrm{Q}_{6}$ for $\delta=0.08$. (c) (Color online) Snapshots showing the spatial positions of small particles in red and big particles in cyan color in the parent structure at temperature $\mathrm{T}^{*}=1.02$ for constant pressure $\mathrm{P}^{*}=10$ and $\delta=0.08$. Note the solid-like structure of the system at $\mathrm{T}^{*}=1.02$. (d) Snapshot shows parent structure at $\mathrm{T}^{*}=1.06$ for constant pressure $\mathrm{P}^{*}=10$ and $\delta=0.08$. Note that figure $5(\mathrm{~d})$ depicts amorphous/glass like formation at $\mathrm{T}^{*}=1.06$. 
Following the definition, a vector $r_{i j}$ pointing from a given molecule (i) to one of its nearest neighbors ( $\mathrm{j}$ ) is denoted as a "bond." For each bond one can define a quantity

$$
q_{l m}\left(r_{i j}\right)=\left(1 / n_{b}\right) \sum_{i} Y_{l m}\left[\theta\left(r_{i j}\right), \phi\left(r_{i j}\right)\right],
$$

where $Y_{l m}\left[\theta\left(r_{i j}\right), \phi\left(r_{i j}\right)\right]$ is spherical harmonics and $\mathrm{n}_{\mathrm{b}}$ is the number of bonds. $\theta\left(r_{i j}\right)$ and $\phi\left(r_{i j}\right)$ are the polar and azimuthal angles of vector $r_{i j}$ respectively with respect to an arbitrary reference frame. The global bond order is obtained by averaging over all bonds in the system,

$$
\bar{Q}_{l m}=\frac{1}{n_{b}} \sum_{\text {bonds }} q_{l m}\left(r_{i j}\right) .
$$

To construct a rotationally invariant local order parameter, one can define $q_{l}$ as,

$$
q_{l}=\left[\frac{4 \pi}{2 l+1} \sum_{m=-l}^{m=l}\left|q_{l m}\right|^{2}\right]^{1 / 2} .
$$

In fcc lattice the orientational order is characterized by six fold symmetry that corresponds to $1=6$, and global bond order, $\mathrm{Q}_{6}=0.5745$ for perfect fcc. ${ }^{55}$

We compute global bond order parameter $\left(\mathrm{Q}_{6}\right)$ as shown in figure 5(b). It is evident from the result that at constant pressure $\mathrm{P}^{*}=10$ and with increasing temperature $\mathrm{T}^{*}$, the value of $\mathrm{Q}_{6}$ decreases gradually, as indicated by figure 5(b). Interestingly, $\mathrm{Q}_{6}$ shows a sharp structural transition at $\mathrm{T}^{*}=1.02$ for $\delta=0.08$. Then, at $\mathrm{T}^{*}=1.06$ for $\delta=0.08$, the crystalline phase disappears completely and enters into the liquid phase.

Figure 5(c) shows snapshots depicting the spatial positions of small particles in red and big particles in cyan color at constant $\mathrm{P}^{*}=10$ and $\delta=0.08$ for temperature $\mathrm{T}^{*}=1.02$ (ending of the upper solid branch as in figure 5(a)) where the solid-like structure is evident. Figure 5(d) shows snapshots at constant $\mathrm{P}^{*}=10$ and $\delta=0.08$ for temperature $\mathrm{T}^{*}=1.06$ (starting of the lower liquid branch as in figure 5(a)) where amorphous/glass like structure is clearly visible.

\subsection{Explanation of the existence of terminal poly- dispersity from bifurcation diagram analysis and Hansen-Verlet rule of freezing}

3.5a Theoretical background: Non-linear integral equation theory of freezing: Integral equation based theoretical analysis of freezing transition has been a subject of great interest for many years. The most successful theory of freezing, the Ramakrishnan-Yussouff theory $^{32,33}$ is also based, at the core, on integral equations that relate the inhomogeneous single particle density to two particle direct correlation function. This theory employs an expansion of density in a Fourier series where order parameters are the density evaluated at the reciprocal lattice vector components. The resulting equations are solved along with the thermodynamics conditions of equality of chemical potential and equality of pressure.

In addition, enormous numbers of theoretical approaches are used to describe the freezing transition directly in terms of the bifurcation of solutions for single density from the homogeneous liquid branch to inhomogeneous branch. This approach also applies essentially the same integral equation that relates the inhomogeneous singlet density to the pair direct correlation function..$^{3,10,20,22-24,61,62}$ Interestingly, a terminal point in the bifurcation diagram is achieved where the density of the liquid phase is close to the dense random close packed state of the hard sphere liquid and the density of the solid phase is close to crystal closed packed values. ${ }^{20}$ This point can be interpreted as the signature of the end of possible compression in the system.

In the elegant approach of the theory of freezing by Ramakrishnan and Yussouff, ${ }^{32,33}$ thermodynamic potential of the system is computed as a function of the order parameters proportionl to the periodic lattice components of the singlet density. This theory employs a nearly exact expression for inhomogeneous density field derived from the density functional theory of statistical mechanics. Free energy is expressed in terms of density fluctuations around the bulk liquid density with n-particle direct correlation functions as the expansion coefficients, as elaborated below.

In accordance with the density functional theory of freezing, the density of inhomogeneous solid can be expressed in terms of order parameters $\phi_{0}$ and $\left\{\phi_{\vec{G}}\right\}$ in the following fashion

$$
\rho_{s}(\vec{R})=\rho_{l}\left(1+\phi_{0}\right)+\rho_{l} \sum_{\vec{G}} \phi_{\vec{G}} \exp (i \vec{G} \cdot \vec{R}),
$$

where $\rho_{s}(\vec{R})$ is the inhomogeneous solid density, $\rho_{l}$ is the liquid density, $\{\vec{G}\}$ is the set of reciprocal lattice vectors of the chosen lattice and $\phi_{0}$ is the fractional density difference between solid and liquid phases.

The order parameters $\phi_{0}$ and $\left\{\phi_{\vec{G}}\right\}$ are the expansion coefficients of the inhomogeneous solid density, defined quantitatively below. These quantities can be quantified in the following fashion,

$$
\rho_{S}=\frac{1}{\Delta} \int_{\Delta} \rho_{S}(\vec{R}) d \vec{R}
$$

where integration is over the lattice cell of volume $\Delta$. 
Structural order parameters $\phi_{\vec{G}}$ can be written as,

$$
\phi_{\vec{G}}=\frac{1}{\Delta} \int \frac{\Delta \rho_{S}(\vec{R})}{\rho_{l}} e^{-i \vec{G} \cdot \vec{R}} d \vec{R},
$$

where $\Delta \rho_{S}(\vec{R})$ is the density difference $\rho_{S}(\vec{R})-\rho_{l}$. Note that eq. (10) is the straightforward consequence of eq. (8) as $\phi_{\vec{G}}$ can be obtained by taking the Fourier transform of $\Delta \rho_{s}(\vec{R})$.

One can introduce a scaling transformation that brings out an interesting generality of the freezing theory as,

$$
\psi_{\vec{G}}=\frac{\rho_{l}}{\rho_{s}} \phi_{\vec{G}},
$$

and

$$
\lambda_{\vec{G}}=\rho_{s} \tilde{c}_{2}(\vec{G}),
$$

with $\tilde{c}_{2}(\vec{G})=\int d R c_{2}(R) e^{i \vec{G} \cdot \vec{R}}$, where $\tilde{c}_{2}(\vec{G})$ is the Fourier transform of the pair direct correlation function, evaluated at the reciprocal lattice vector $\vec{G}$.

In order to make subsequent connection with the liquid state we use,

$$
\begin{aligned}
\lambda_{\vec{G}} & =\rho_{s} \tilde{c}_{2}(\vec{G}) \\
& =\frac{\rho_{s}}{\rho_{l}}\left[\rho_{l} \tilde{c}_{2}(\vec{G})\right] \\
& =\frac{\rho_{s}}{\rho_{l}}\left[1-\frac{1}{S(\vec{G})}\right] .
\end{aligned}
$$

Here $S(\vec{G})$ is the value of first peak maximum of the liquid structure factor. $\lambda_{\vec{G}}$ and $\psi_{\vec{G}}$ are both dimensionless quantities. The scaling transformation allows one to solve $\psi_{\vec{G}}$ as a function of $\lambda_{\vec{G}}$.

The above transformations lead to the following expression $\psi_{\vec{G}_{n}}$,

$$
\psi_{\vec{G}_{n}}=\frac{\int_{\Delta} d \vec{R}_{1} \xi_{\vec{G}_{n}}\left(\vec{R}_{1}\right) \exp \left[\sum_{\vec{G}} \psi_{\vec{G}} \lambda_{\vec{G}} \xi_{\vec{G}}\left(\vec{R}_{1}\right)\right]}{\int_{\Delta} d \vec{R}_{1} \exp \left[\sum_{\vec{G}} \psi_{\vec{G}} \lambda_{\vec{G}} \xi_{\vec{G}}\left(\vec{R}_{1}\right)\right]},
$$

where $\vec{G}_{n}$ is the n-th reciprocal lattice vector, $\xi_{\vec{G}_{n}}$ is the periodic density wave corresponds to the set of $\{\vec{G}\}$ that has the same magnitude, with

$$
\xi_{G_{\alpha}}(R)=\sum_{\left\{G_{\alpha}\right\}} e^{i G_{\alpha} \cdot \vec{R}} .
$$

Equation (13) and hence the scaled phase diagram, as shown in figure 6(a), has been obtained under the assumption of equality of chemical potential between the liquid and solid phases. Equation (13) does not require any information about direct correlation function or density of the liquid and solid phases. Only the lattice type needs to be specified. Thus, the bifurcation diagram generated by eq. (13) can be regarded as 'universal' for a chosen lattice, as noted by Rice et al. ${ }^{20}$ At the bifurcation point, for a certain value of $\lambda_{\vec{G}}$, there is a sudden appearance of the multiple values of $\psi_{\vec{G}}$, indicative of the appearance of a new phase.

The free energy change, as a function of the order parameters, has been given by Ramakrishnan-Yussouff ${ }^{32}$ and by Haymet-Oxtoby. ${ }^{37}$ Using the density functional theory, one can obtain the expression of the grand canonical potential difference between solid and liquid as,

$$
\begin{gathered}
\Delta \Omega=\left(\rho_{l} c_{0}-1\right) \phi_{0}+\frac{1}{2} c_{0} \rho_{l} \phi_{0}^{2}+\frac{1}{2} \sum_{\vec{G}} \tilde{c}_{2}(\vec{G}) \phi_{\vec{G}}^{2}, \\
c_{0} \equiv \tilde{c}_{2}(0) .
\end{gathered}
$$

For the calculation of phase transition parameters, Eq. (15) needs to be supplied by the equality of thermodynamic potential as $\Delta \Omega=\Omega_{s}-\Omega_{l}=0$. Since $\phi_{0}$ and $\phi_{\vec{G}}$ are all positive, $\Delta \Omega$ can be zero only if $\left(\rho_{l} c_{0}-1\right) \phi_{0}+\frac{1}{2} c_{0} \rho_{l} \phi_{0}^{2}$ is compensated by $\frac{1}{2} \widetilde{c}_{2}(\vec{G}) \phi_{\vec{G}}^{2}$. The above expressions are all for one component liquid. However, as shown by Chaudhuri et al. ${ }^{34}$, the same expression may be used with an effective $c_{0}$ and effective $\tilde{c}_{2}(\vec{G})$. The order parameters themselves can be determined by a set of non-linear equations, as shown by Rice et al. ${ }^{61,62}$

Our present study on Lennard-Jones system leads to the conclusion that the system of nonlinear integral equations, used to describe the transition, displays two bifurcation points. The scaled phase diagram with scaled variables is shown in figure 6(a). Note that the diagram shows a curious character. The scaled phase diagram is just like a van der Waals loop with two terminal points (denoted by blue and black dots, as shown in figure 6(a)) that may be identified as the two spinodal points of the solid-liquid phase transition. The first turn (near blue dot) is the one that corresponds to the limit of stability of the solid, as discussed here, and is connected to terminal solid density. The second turn (near black dot) at larger density for $\lambda_{\vec{G}}=1$ corresponds to the limit of stability of the liquid as discussed earlier by Rice et $a l .{ }^{20}$ and even earlier by Lovett. ${ }^{10}$ Maxwell tie line (given by $\Delta \Omega=0$ ) is constructed to find coexistence under a given thermodynamic condition. This enforces the additional thermodynamic condition of equality of pressure between the fluid and the solid branches. Coexistence is found by imposing equality of grand thermodynamic potential. 

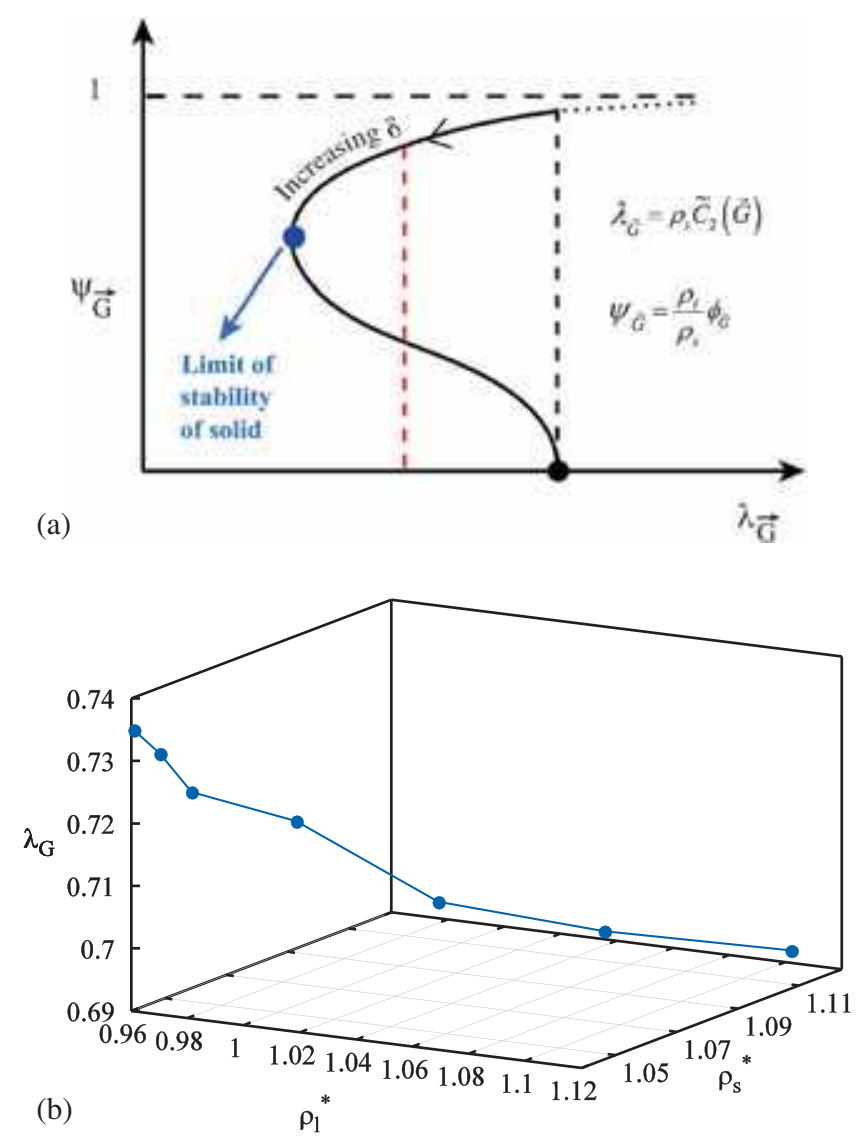

Figure 6. (a) Two order parameter $\left(\phi_{0}\right.$ and $\left.\phi_{\vec{G}}\right)$ scaled phase diagram plotted in scaled units. Scaling transformation is given by $\psi_{\vec{G}}=\frac{\rho_{l}}{\rho_{s}} \phi_{\vec{G}}$ and $\lambda_{\vec{G}}=\rho_{s} \tilde{c}_{2}(\vec{G})$, where $\tilde{c}_{2}(\vec{G})$ is the Fourier transform of the pair direct correlation function evaluated at the reciprocal lattice vector $\vec{G}$. The two end points (denoted by blue and black dots) signify two limits of stability. The first terminal point (denoted by blue dot) gives the limit of stability of the solid, as discussed here and is connected to terminal solid density. The second terminal point (denoted by black dot) at $\lambda_{\vec{G}}=1$ gives the limit of stability of liquid as discussed earlier by Rice et al., ${ }^{20}$ and even earlier by Lovett. ${ }^{10}$ (b) Variation of scaled order parameter $\left(\lambda_{\vec{G}}\right)$ in the phase diagram with coexistence density of solid $\left(\rho_{s}^{*}\right)$ and liquid $\left(\rho_{l}^{*}\right)$ at constant $\mathrm{T}^{*}=1$. Note that the value of the scaled order parameter $\left(\lambda_{\vec{G}}\right)$ decreases as both the coexistence density of solid $\left(\rho_{s}^{*}\right)$ and that of liquid $\left(\rho_{l}^{*}\right)$ increase.

Note that the theory used to obtain the scaled phase diagram with scaled variables, as shown in figure 6(a), does not contain the polydispersity explicitly, but it is assumed to hold good even for a polydisperse system. The influence of polydispersity is recovered on the top of the treatment via its effect on the order parameters $\lambda_{\vec{G}}$ and $\psi_{\vec{G}}$, whose motion is then followed with increasing $\delta$, denoted by black arrow along the curve, as shown in figure 6(a).
Along $X$ axis, $\lambda_{\vec{G}}$ can correspond to multitudes of $\rho_{S}$ and $\rho_{l}$ values within the region of scaled phase diagram. This is because $\lambda_{\vec{G}}$ depends intricately on both $\rho_{S}$ and $\rho_{l}$ values through direct correlation function which is evaluated with $\rho_{l}$, but at the reciprocal lattice vector $\vec{G}$ which depends on the solid density $\rho_{S}$. Equality of pressure for grand canonical ensemble picks out unique values of $\rho_{S}$ and $\rho_{l}$. As $\delta$ increases, both of the changes in $\lambda_{\vec{G}}$ and $\psi_{\vec{G}}$ values drive the system to the turning point/spinodal point, denoted by blue dot on the curve, as shown in figure 6(a). Hence the motion of the order parameters $\lambda_{\vec{G}}$ and $\psi_{\vec{G}}$, induced by the increase in $\delta$, drives the system towards disappearance of the ordered solid where no liquid-solid transition is possible.

3.5b Relationship among scaled order parameter $\left(\lambda_{\vec{G}}\right)$ in the scaled phase diagram and coexistence densities of solid and liquid: With the increase in polydispersity indices, the coexistence density of the solid $\left(\rho_{s}^{*}\right)$ approaches to that of the liquid $\left(\rho_{l}^{*}\right)$ and the value of the direct correlation function at its first peak decreases. Note that by means of eq. (12), we obtain the values of the direct correlation function and order parameter $\left(\lambda_{\vec{G}}\right)$. Corresponding to a particular temperature and polydispersity index, we find the value of first peak maximum of the liquid structure factor $\left[\mathrm{S}\left(\mathrm{k}_{\mathrm{M}}\right)_{\text {liquid }}\right]$ obtained from numerical simulations and use the value of $\left[\mathrm{S}\left(\mathrm{k}_{\mathrm{M}}\right)_{\text {liquid }}\right]$ in place of $S(\vec{G})$ of eq. (12) as because these two values are almost comparable [more details given in Section $3.5 \mathrm{c}]$. For a constant temperature $\mathrm{T}^{*}=1$, the value of the order parameter $\left(\lambda_{\vec{G}}\right)$ in the scaled phase diagram decreases with the increase in the coexistence density of solid $\left(\rho_{s}^{*}\right)$ and that of liquid $\left(\rho_{l}^{*}\right)$ as shown in figure 6(b).

This case is similar to the one when one moves toward left along the upper branch in the scaled phase diagram, denoted by black arrow along the curve, as shown in figure 6(a). Since no transition is possible beyond the spinodal point, this point can be identified with the instability point of Lindemann melting criterion.

3.5c Hansen-Verlet rule of crystallization in freezing of Lennard-Jones polydisperse system: In our earlier work ${ }^{1}$ we have shown the solid-liquid coexistence lines for different polydispersity from free energy calculation using umbrella sampling. In the present work, along the coexistence line for a particular co-existence volume fraction $\Phi$, we calculate the structure factor of the liquid phase against polydispersity index $\delta$ at constant temperature $\mathrm{T}^{*}=1$. The values of the coexistence pressure are given in table 2 . The first peak maxima of the 
liquid structure factor $\mathrm{S}\left(\mathrm{k}_{\mathrm{M}}\right)_{\text {liquid }}$ for different polydispersity indices are shown in figure 7 . The plot shows that the first peak maximum increases with polydispersity till $\delta=0.08$ and then it starts decreasing with $\delta$. The red dotted line in the figure 7 shows the actual line predicted by the Hansen-Verlet rule of crystallization where the value of the structure factor is exactly 2.85 . The structural transformation happens at polydispersity $\delta=0.095$ as shown by black arrow in figure 7 . Note that at temperature $\mathrm{T}^{*}=1$ and at polydispersity index $\delta=0.10$, the value of the structure factor of the liquid phase is 2.74 which is below the value of 2.85 needed for freezing as predicted by structure factor analysis. This means that the freezing is not possible at $\delta=0.10$ and the system is in the liquid phase for $\delta=0.10$.

\subsection{Discussions}

In our present study we are interested in melting of the solid due to increase in polydispersity, as elaborated by the IS analysis. As the polydispersity increases, several things occur that determine the position of the coexistence. Freezing shifts continuously to the higher volume fraction (and pressure). The density of the solid at coexistence approaches that of the liquid and as a result fractional density change $\left(\phi_{0}\right)$ decreases.

Also, with the increase in polydispersity index, the crystalline order of the solid decreases progressively which is reflected in the lower value of the order parameters. The value of the ordinate (that gives scaled order parameter, $\psi_{\vec{G}}$ ) in the scaled phase diagram decreases and at the same time, value of the abscissa also decreases. All these lead the system towards the first terminal point which can thus be identified as the limit of stability of the polydisperse solid with respect to the liquid. The results obtained for the melting transition parameters (at $\mathrm{T}^{*}=1$ ) of the Lennard-Jones polydisperse solids are shown in table 2 .

As evident from table 2, also indicated in figure 6(a), with increasing polydispersity index $\delta$, density of liquid at solid-liquid transition increases. This leads to an increase in the value of first peak maximum of the liquid structure factor, $\mathrm{S}\left(\mathrm{k}_{\mathrm{M}}\right)_{\text {liquid }}$. At the same time, value of the scaled order parameter $\left(\lambda_{\vec{G}}\right)$ decreases. This is

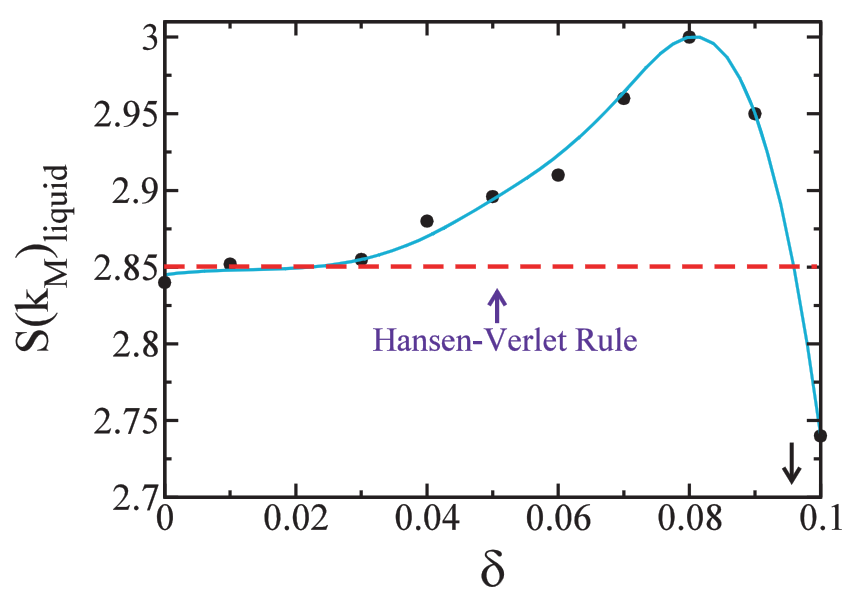

Figure 7. The variations of the first peak maximum of liquid structure factor $\mathrm{S}\left(\mathrm{k}_{\mathrm{M}}\right)_{\text {liquid }}$ with different polydispersity indices $(\delta)$. The structure factor of the liquid phase for different $\delta$ is calculated for the corresponding co-existence volume fraction $\Phi$ at temperature $\mathrm{T}^{*}=1$. The red dotted line signifies the predicted value obtained from Hansen-Verlet rule of crystallization. Please note that black arrow shows the structural transformation at polydispersity $\delta=0.095$ predicted by structure factor analysis that agrees well with all other analyses (inherent structure, bifurcation analysis, Lindemann criterion) presented here.

Table 2. Comparison of values of melting parameters at $\mathrm{T}^{*}=1$. The first column represents value of polydispersity index $(\delta)$ and second column represents corresponding pressure $\left(P^{*}\right)$ at the coexistence of solid and liquid phases. Third and fourth columns represent coexistence density of solid $\left(\rho_{s}^{*}\right)$ and its volume fraction $\left(\phi_{S}\right)$ respectively. Fifth and sixth columns represent coexistence density of liquid $\left(\rho_{l}^{*}\right)$ and its volume fraction $\left(\phi_{l}\right)$ respectively. The value of $\phi_{0}$ represents fractional density change on melting transition. $S\left(\mathbf{k}_{M}\right)_{\text {Liquid }}$ shows the value of the liquid structure factor at the first peak. In the last column $\lambda_{\vec{G}}$ represents the value of the scaled order parameter. Note that $\lambda_{\vec{G}}=\rho_{s} \tilde{c}_{2}(\vec{G})$, where $\tilde{c}_{2}(\vec{G})$ is the Fourier transform of the pair direct correlation function, evaluated at the reciprocal lattice vector $\vec{G}$.

\begin{tabular}{|c|c|c|c|c|c|c|c|c|}
\hline$\delta$ & $P^{*}$ & $\rho_{s}^{*}$ & $\phi_{S}$ & $\rho_{l}^{*}$ & $\phi_{l}$ & $\phi_{0}$ & $S\left(\mathbf{k}_{M}\right)_{\text {Liquid }}$ & $\lambda_{\vec{G}}$ \\
\hline 0.0 & 6.58 & 1.039 & 0.544 & 0.961 & 0.503 & 0.075 & 2.839 & 0.735 \\
\hline 0.01 & 6.73 & 1.042 & 0.546 & 0.967 & 0.507 & 0.072 & 2.852 & 0.731 \\
\hline 0.03 & 7.10 & 1.044 & 0.547 & 0.976 & 0.511 & 0.065 & 2.855 & 0.725 \\
\hline 0.04 & 8.28 & 1.058 & 0.554 & 0.998 & 0.523 & 0.058 & 2.879 & 0.719 \\
\hline 0.06 & 10.40 & 1.074 & 0.563 & 1.031 & 0.540 & 0.040 & 2.911 & 0.705 \\
\hline 0.07 & 12.97 & 1.097 & 0.575 & 1.065 & 0.558 & 0.029 & 2.961 & 0.698 \\
\hline 0.08 & 16.30 & 1.124 & 0.588 & 1.102 & 0.577 & 0.019 & 3.001 & 0.692 \\
\hline 0.09 & - & - & & - & - & & 2.949 & - \\
\hline 0.10 & - & - & & - & - & & 2.740 & - \\
\hline
\end{tabular}


the direct consequence of the sharp fall in the fractional density change $\left(\phi_{0}\right)$ on melting-freezing. Interestingly, the value of the polydispersity index (for a constant volume fraction) at which melting is predicted agrees well with Lindemann criteria of atom displacement.

In order to explore the mechanical stability of a metastable solid through inherent structure analysis, the potential energy of the solid must be a minimum. We find that beyond a given polydispersity index (at a constant volume fraction) no such minimum, indicating that amorphous state represents the true ground state of the system.

From the liquid side, we can get an estimate of the transfer of stability from liquid to solid state by (a) Ramakrishnan and Yussouff theory ${ }^{32}$ (b) empirical Hansen-Verlet rule. ${ }^{7}$ Both again give values of liquid and solid density those are in agreement with inherent structure analysis and integral equation based theoretical analysis of liquid-solid transition.

\section{Conclusions}

Let us first summarize the main results. This paper describes an investigation of the effect of polydispersity on the solid-liquid transition of the Lennard-Jones system. To this purpose, the system is studied by numerical simulations at different degree of polydispersity and its behavior is analyzed in three distinct ways, namely, by applying the empirical Lindemann criterion of melting, the inherent structure analysis and the empirical Hansen-Verlet criterion of freezing. The results thus obtained are found to be consistent with one another, both qualitatively and quantitatively, in predicting the existence of a terminal polydispersity, beyond which freezing disappears. We find that before melting, the average inherent structure energy and RMSD of the solid show similar polydispersity dependence including a discontinuity at solid-liquid transition. Lindemann ratio, obtained from RMSD, is found to be strongly dependent on temperature.

The terminal polydispersity has not been studied earlier in the Lennard-Jones polydisperse system using non-linear integral equation theories of freezing. The above finding of the terminal polydispersity is interpreted in terms of the theory of freezing developed by Bagchi, Cerjan, and Rice, ${ }^{20}$ and the conclusion is that the motion of the order parameters induced by the increase of polydispersity also points towards the disappearance of the ordered solid. We discuss how certain features of the scaled phase diagram may be used to explain the terminal polydispersity in polydisperse system. This analysis implies that at high polydispersity, the equality of thermodynamic potential between solid and liquid phases can no longer be established. The liquid state has lower chemical potential and the solid phase is not possible beyond certain polydispersity. This is manifested in the absence of any solution of the non-linear integral equations. The same feature is reflected in the inherent structure analysis - no crystalline solid is obtained in the inherent structure beyond the terminal polydispersity. The amorphous state is the global minimum in the potential energy surface.

\section{Acknowledgements}

We would like to thank Prof. Stuart A. Rice, Prof. Charusita Chakravarty, Prof. Govardhan Reddy for scientific discussions. This work was supported in parts by grants from DST and CSIR (India). B.B. thanks DST for support through J.C. Bose Fellowship.

\section{References}

1. Sarkar S, Biswas R, Santra M and Bagchi B 2013 Phys. Rev. E $\mathbf{8 8} 022104$

2. Abraham S E, Bhattacharrya S M and Bagchi B 2008 Phys. Rev. Lett. 100167801

3. Bagchi B, Cerjan C, Mohanty U and Rice S A 1984 Phys. Rev. B 292857

4. Phan S-E, Russel W B, Zhu J and Chaikin P M 1998 J. Chem. Phys. 1089789

5. Lindemann F A 1910 Phys. Z. 11609

6. Wales D J 2003 In Energy landscapes (Cambridge: Cambridge University Press)

7. Hansen J-P and Verlet L 1969 Phys. Rev. 184151

8. Kirkwood J G and Monroe E 1941 J. Chem. Phys. 9514

9. Kozak J J, Rice S A and Weeks J D 1971 Physica 54573

10. Lovett R 1977 J. Chem. Phys. 661225

11. Munakata T 1977 J. Phys. Soc. Jpn. 431723

12. Munakata T 1978 J. Phys. Soc. Jpn. 45749

13. Lacks D J and Wienhoff J R 1999 J. Chem. Phys. 111 398

14. Kofke D A and Bolhuis P G 1999 Phys. Rev. E 59618

15. Williams S R, Snook I K and Megen van W 2001 Phys. Rev. E 64021506

16. Auer S and Frenkel D 2001 Nature 413711

17. Hansen J-P and McDonald I R 2006 In Theory of Simple Liquids 3rd Edition (San Diego: Academic Press)

18. Maeda K, Matsuoka W, Fuse T, Fukui K and Hirota S 2003 J. Mol. Liq. 1021

19. Bolhuis P G and Kofke D A 1996 Phys. Rev. E 54634

20. Bagchi B, Cerjan C and Rice S A 1983 J. Chem. Phys. 795595

21. Bagchi B, Cerjan C and Rice S A 1983 J. Chem. Phys. 796222 
22. Bagchi B, Cerjan C and Rice S A 1983 Phys. Rev. B 28 6411

23. Radloff P L, Bagchi B, Cerjan C and Rice S A 1984 J. Chem. Phys. 811406

24. Lovett R and Buff F P 1980 J. Chem. Phys. 722425

25. Sarkar S and Bagchi B 2011 Phys. Rev. E 83031506

26. Chakraborty S N and Chakravarty C 2007 Phys. Rev. E 76011201

27. Singh M, Agarwal M, Dhabal D and Chakravarty C 2012 J. Chem. Phys. 137024508

28. Ingebrigtsen T S, Schrøder T B and Dyre J C 2012 Phys. Rev. X 2011011

29. Fantoni R, Gazzillo D, Giacometti A and Sollich P 2006 J. Chem. Phys. 125164504

30. Tokuyama M and Terada Y 2005 J. Chem. Phys. B 109 21357

31. Bartlett P and Warren P B 1999 Phys. Rev. Lett. 821979

32. Ramakrishnan T V and Yussouff M 1979 Phys. Rev. B 192775

33. Ramakrishnan T V 1982 Phys. Rev. Lett. 48541

34. Chaudhuri P, Karmakar S, Dasgupta C, Krishnamurthy H R and Sood A K 2005 Phys. Rev. Lett. 95248301

35. Singh Y, Stoessel J P and Wolynes P G 1985 Phys. Rev. Lett. 541059

36. Corti D S, Debenedetti P G, Sastry S and Stillinger F H 1997 Phys. Rev. E 555522

37. Haymet A D J and Oxtoby D W 1981 J. Chem. Phys. 74 2559

38. Haymet A D J and Oxtoby D W 1986 J. Chem. Phys. 84 1769

39. Oxtoby D W and Haymet A D J 1982 J. Chem. Phys. 76 6262

40. Ramakrishnan T V and Sengupta S 1993 Density wave theory of freezing and of interfaces. In: Indo-US workshop on Interfaces, 1993, pp. 33-49

41. Underwood S M, Taylor J R and Megen van W 1994 Langmuir 103550

42. Pusey P 1987 J. Phys. 48709
43. Pusey P 1991 In Les Houches, Session LI, Liquids, Freezing and Glass Transitions, NATO Advanced Study Institute, Series B: Physics J P Hansen, D Levesque and J Zinn-Justin (Eds.) (Amsterdam: Elsevier) Ch. 10

44. Chakravarty C, Debenedetti P G and Stillinger F H 2007 J. Chem. Phys. 126204508

45. Agrawal R and Kofke D A 1995 Mol. Phys. 8543

46. Schneider T, Brout R, Thomas H and Feder J 1970 Phys. Rev. Lett. 251423

47. Schneider T 1971 Phys. Rev. A 32145

48. Yang A J M, Fleming P D and Gibbs J H 1976 J. Chem. Phys. 643732

49. Löwen H, Palberg T and Simon R 1993 Phys. Rev. Lett. 701557

50. Löwen H and Hoffmann G P 1999 Phys. Rev. E 603009

51. Stoessel J P and Wolynes P G 1984 J. Chem. Phys. 80 4502

52. Langer J S 2013 Phys. Rev. E 88012122

53. Leocmach M, Russo J and Tanaka H 2013 J. Chem. Phys. 138 12A536

54. Lovett R, Mou C Y and Buff F P 1976 J. Chem. Phys. 65570

55. Press W, Flannery B, Teukolsky S and Vetterling W 1992 In Numerical Recipes in Fortran 77: The Art of Scientific Computing (UK: Cambridge University Press)

56. Nelson D R 2002 In Defects and geometry in condensed matter physics (UK: Cambridge University Press)

57. Landau L D and Lifshitz E M 1981 In Theory of Elasticity Second revised and enlarger edition (Oxford: Pergamon press)

58. Jarić M V and Mohanty U 1988 Phys. Rev. B 374441

59. Lipkin M D, Rice S A and Mohanty U 1985 J. Chem. Phys. 82472

60. Steinhardt P J, Nelson D R and Ronchetti M 1983 Phys. Rev. B 28784

61. Xu X and Rice S A 2008 Phys. Rev. E 78011602

62. Xu X and Rice S A 2011 Phys. Rev. E 83021120 